BORN-OPEN DATA FOR JSPSYCH

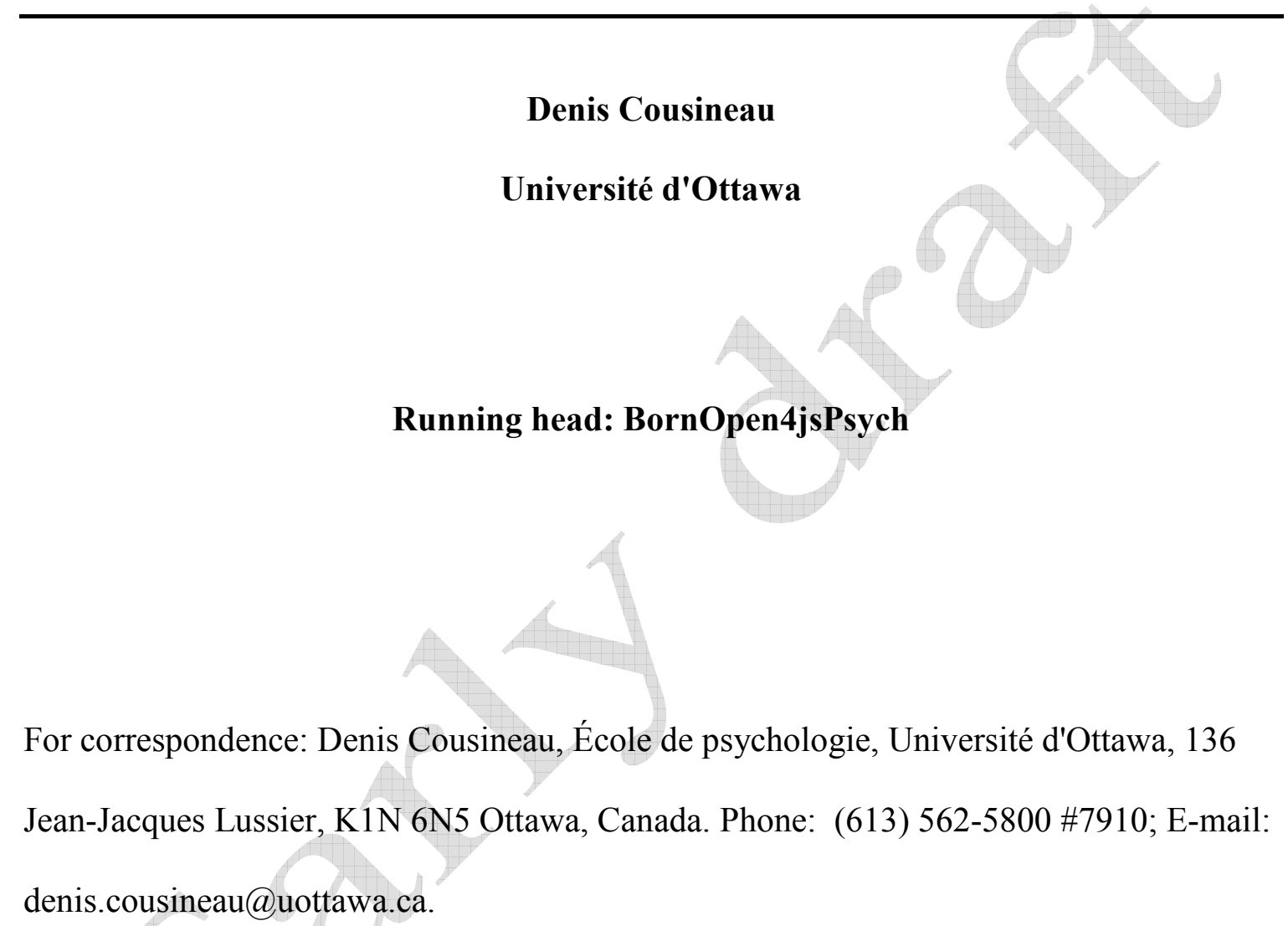




\begin{abstract}
Born-Open Data is a framework whereby experiments are conceived from the start as making the data openly accessible. This framework was first described in Rouder, 2016, and an actual implementation for E-Prime 2.10 was provided by Cousineau, 2020. Herein, we provide an implementation for jsPsych. It consist in a plugin for jsPsych which can be inserted anywhere in the experiment's timeline and a function called upon completion of the experiment. The data are then uploaded transparently and automatically to a GitHub repository where they are immediately and openly accessible to the research community.
\end{abstract}

Keywords: Born-Open data; open-access data; repositories; 


\section{Born-Open Data for jsPsych}

\section{$\underline{\text { Introduction }}$}

Born-Open Data is an approach whereby experiments are designed and programmed in a way that the data are directly accessible to the research community. The process must be as direct as possible (no filters) and as transparent as possible. First proposed by Rouder in 2016, it stem from open science initiatives meant to promote better scientific practices (e.g., Simmons et al., 2011). One implementation for E-Prime was described in Cousineau (2020) and used in Maxcey, Shiffrin, Cousineau and Atkinson (submitted).

To be useful, an implementation must be easy to use. An ideal implementation should consist of one or a few commands added to the script of an experiment, from which everything else is done transparently. Further, it should be easy to disable these commands (placing them as comments in the script) while performing tests and decommenting them when beta testing is completed without impact on the rest of the script.

Herein, we describe an implementation for jsPsych experiments. This implementation was prompted by the COVID pandemic. Prior to this, our experiments were run in a laboratory setting, with computers that were specifically configured to handle experiments. With the advent of the pandemic, many experiments were moved to online environment running on personal computers which may not have specialized software. This added a level of complexity to the present endeavor. Indeed, the two 
previous implementations of Born-Open data used GitHub as the container (the repository) and git as the protocol to interact with the container (Chacon \& Straub, 2014). However, whereas it is very easy to install git on a laboratory computer, we cannot expect that it will be present on a participant's computer. Therefore, all repository manipulations are now performed by a server, a computer accessible through the web.

We chose jsPsych as the platform for programming the experiments because it was specifically created for that purpose and is openly and freely accessible (de Leew, 2015). It is also fairly easy to program experiments with this platform (which extends JavaScript capabilities with dedicated functions called plugins), and the developers' team provides timely support. Bridges et al. (2020), among others, have documented the measurement precision of j s P sych and other online programming environments for psychology experiment with respect to collecting response times and presenting visual stimuli. At the time of this writing, js ssych version 6.1 was used, but a version 7 is scheduled to be released soon.

In what follows, we present the plugin BornOpen4jsPsych-Initialize and an accompanying function that triggers the upload of the data. Next, we describe the general process underlying the plugin. The interested reader can try a dummy experiment located at tqmp.org/BornOpen4jsPsych/testExperiment/ (URL are case-sensitive). The participant ID "e1W2q1" (case-sensitive as well) is allowed to run session 1 indefinitely. The responses collected are transferred to the following repository github.com/VIC-Laboratory-ExperimentalData/Test in the folder called rawdata within seconds. 


\section{$\underline{\text { The plugin }}$}

The BornOpen4jsPsych plugin is contained in a JavaScript file called Bornopen4jsPsych-Initialize.js. This file must be placed in the folder where the experiment resides (typically, the experiment is contained in an HTML page). Inside the experiment, the following lines of code are needed to load the plugin and its style sheet:

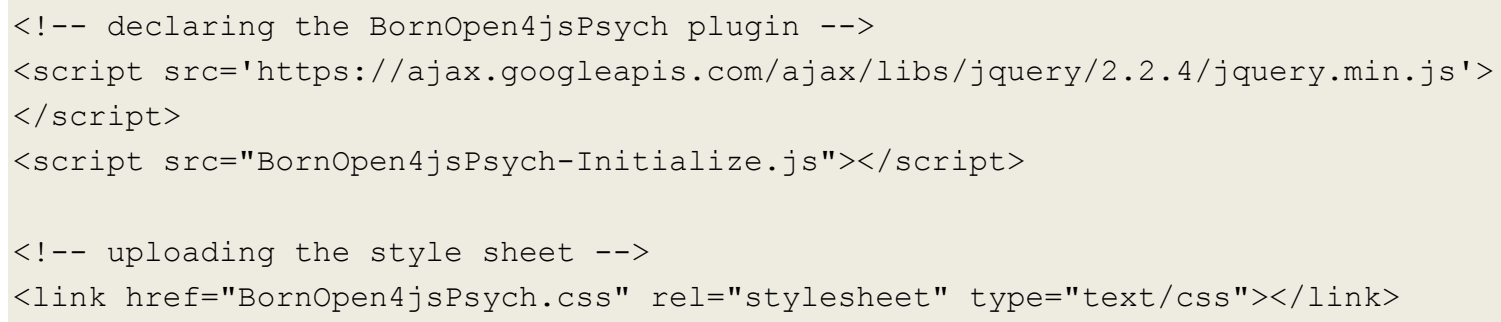

Because the Bornopen $4 \mathrm{j}$ s Psych plugin requires extensions, these must be loaded as well (the jquery extensions, available from Google).

In the experiment's timeline, an event must be inserted which initializes the BornOpen4jsPsych capabilities. The simplest description of the event is:

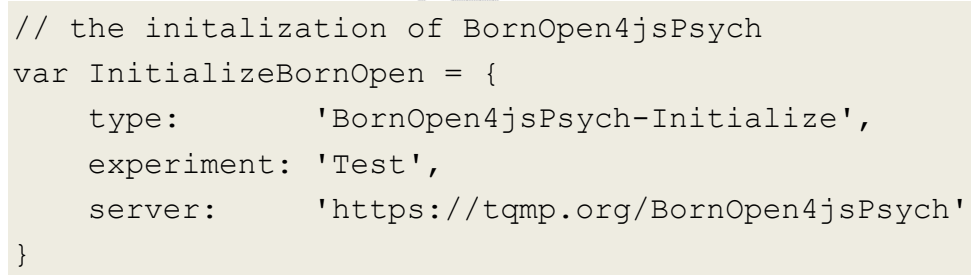

This event results in a slide being shown in the experiment; a screen captures is seen in

Figure 1. This event is contained in a variable, here named InitializeBornopen (the name is arbitrary). It has the following attributes:

- type: a mandatory jsPsych attribute describing the plugin to be used. Here the name Bornopen 4 js Pych-Initialize corresponds to the JavaScript file 
containing the description of the plugin, BornOpen 4 jsPsychInitialize.js.

- experiment: This mandatory attribute defines the name of the experiment. The experiment must be defined on that server (see below). Here, the experiment is called Test (case sensitive).

- $\quad$ server: This mandatory attribute indicates which server (along with any path if needed) holds the relevant server-side programs to process the born-open data requests.

In addition to these mandatory information, two optional attributes can be provided:

- session: If the experiment is composed of a single session, it is possible to fix the session number within the event, with, for example, session : "1". If a session identifier is given, that information will not be requested from the "Identification" slide shown in Figure 1.

- subject: Likewise, if it is not necessary to have an identifier for each participant. It is possible to pre-specify a single subject ID in the initialization (or fetch the subject ID from other means).

When both subject and session are pre-specified, the "Identification" slide is not shown at all.

This step will initialize interval variables. It will also verify that this subject ID is allowed to perform that session ID by consulting a list of invited participants on the 
server. If not found in the invitations, the button "Begin the experiment" remains disabled and the experiment cannot proceed any further.

\section{Triggering the upload}

The above slide allows a participant to continue the experiment. However, it does not trigger the upload of the data. To that end, it is necessary to call the function called Bornopen 4 jsPsychSave (made available when the plugin is loaded). This function can be used anywhere, although the best time is upon completion of experiment, using the on_finish trigger of the jsPsych.init () main function. For example, the following inserted into the jsPsych. init () will trigger an upload of all the data collected in the experiment:

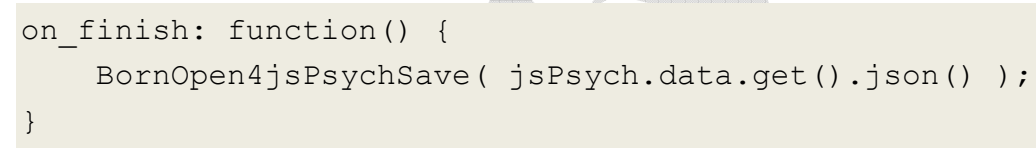

The "get () . json ()" method of the jsPsych. data collects all the data into a JavaScript Object Notation format (the only format accepted by BornOpen 4 jsPsych). Note that all the data are collected, including the time to respond to the welcome screen. If desired, it is possible to filter the data and save only that subset of data. Unless you are familiar with filtering in jsPsych, I would recommend saving all the data and filtering at the data analysis stage. 


\section{The process behind BornOpen 4 jsPsych}

The events triggered by BornOpen 4 js s sych can be subdivided into two stages, the first stage occurring when the initialization plugin is called, the second when the upload function is called. The whole process is schematized in Figure 2.

\section{When initialization is performed:}

The experiment name, subject ID and session ID are sent over the web on the server specified in the plugin. There, a PHP program receives that information through a POST protocol and determines whether that experiment exists. The experiment exists if there is a subfolder of that name. Next, within that subfolder, it consults a file called _invitations.txt to see if the duo subject ID/session ID is present and can still run that session. If that session has already been run, a "done" indication is found next to the pair of information. If the participant is allowed to run that session, an indication once, a number of remaining runs or inf (standing for infinite) is found in the file.

The code for this program is available on github.com/dcousin3/BornOpenData4jsPsych, in the file _checkExperimentSubjectSession.php).

For the Test experiment accompanying this text, the file invitations.txt is accessible through the URL obtained by concatenating the server, the experiment's name and _invitations.txt, separated with slashes, e.g., for the current demo: 
In this demo, a single participant is allowed to run session 1 an infinite amount of times. The email address will be used on the second stage.

When the participant's subject and session IDs are valid, the button "Begin the experiment" is enabled and the participant can complete the subsequent events in the timeline.

\section{When uploading is performed:}

When the function BornOpen $4 \mathrm{j}$ sPsychSave is called, the data are sent over to the server along with the subject and session ID, as initialized by the plugin. There, the server, through a PHP program (available as

_saveExperimentSubjectSession.php on

github.com/dcousin3/BornOpenData4jsPsych) will consult in the experiment's folder a file called_owner.txt. This file contains the experimenter's name and email (on distinct lines). It also contains the GitHub repository and its owner. You can consult the _owner. txt file for the present illustration by concatenating the server's location, experiment name, and "_owner.txt", i.e.:

https://tqmp.org/BornOpen4jsPsych/Test/ owner.txt

With this information, it will add the current data file into the repository found at

https://github.com/OWNER/REPOSITORY

under a folder called "rawdata". In the present illustration, this represents 
The credentials for logging into GitHub must be stored on the server, typically with a Secured Shell (SSH) protocol (see git and ssh documentations). The user whose credentials are kept on the server does not have to be the owner/creator of the repository but must have the relevant privileges to clone and push data on that repository.

Each data file will be named by concatenating the experiment's name, subject ID, session ID, separated by a dash (and a character string starting with " $b$ " if that subject completed that session more than once). The file will be saved as a comma-separated text file, a format accessible to all software/programming languages.

If a file subjectsLog. txt if found in the repository, an additional line with the participant's ID, session ID, date and time will be appended at the end of that file.

It then sends an email to the experimenter (containing a copy of the data) as well as acknowledge of reception to the participant (whose email was in the fourth column of the file_invitations.txt). Consequently, any time you test the present application, the owner(denis.cousineau@uottawa; that is, me) and the participant (denis.couisineau@yopmail.com, a password-free email account) will receive and email.

Finally, a copy of the data file is also kept on the server. Although this is not necessary (the owner receives a copy by email and there is a copy on GitHub), I chose not to remove it for extra security. If you have limited disk space on your server, consider removing these extra copies from times to times.

\section{$\underline{\text { Prerequisite }}$}

In sum, to implement this BornOpen $4 \mathrm{j}$ s Psych protocol, you need, regarding the jsPsych experiment, to 
1- copy the file BornOpen4jsPsych.js and Bornopen4jsPsych. css where your experiment's HTML file is located (it can be on the web or on local files);

2- in the experiment, add a Bornopen4jsPsych-Initialize event early in your timeline and a call to the function Bornopen 4 jsPsychSave at the end;

On a server to which you have access, you need to

3- copy the two PHP scripts which run stage-one and stage-two processes (namely _checkExperimentSubjectSession.php and _saveExperimentSubjectSession.php; both are available on github.com/dcousin3/BornOpenData4jsPsych);

4- on that server, for each experiment you want to run, create a subfolder with the experiment's name and store in this folder the invitations and owner's information (file_invitations.txt and_owner.txt). I chose to proceed with IDs stored in an invitation file to control who runs the experiment and how many times they can run it.

These last two files are presently openly accessible to anyone who knows where to direct a browser. It is possible to have them inaccessible to the public by adding to the server's . htaccess file the following line 
With this line, any folder where a file with the name index. html cannot be found will be forbidden (error 403: access denied). Other solutions are also possible to hide files on a server.

The reader will also find on github.com/dcousin3/BornOpenData4jsPsych an archive file (testExperiment.zip) which contains the demonstration experiment with all its accompanying files located at https://tqmp.org/BornOpen4jsPsych/testExperiment/

\section{Conclusion}

I chose to develop the BornOpen 4 j s P sych system around an invitation list. That choice was made to control who can run the experiment. Without the proper sybject ID/session ID pair, the experiment cannot be run. In addition, is it possible to control the number of times a participant can re-run the experiment (from a single access, to multiple access, or even infinite access). This control is necessary in experiments whose focus is on training effects. 


\section{References}

Bridges, D., Pitiot, A., MacAskill, M., R., \& Peirce, J. W. (2020). The timing megastudy: comparing a range of experiment generators, both lab-based and online. PeerJ, 8, e9414-1-e9414-29. doi: 10.7717/peerj.9414

Chacon, S., \& Straub, B. (2014). Pro Git (2nd edition). Washington: APress.

Cousineau, D. (2020, September 11). Born-Open Data for E-Prime. doi: 10.31234/osf.io/kyuvs

de Leeuw, J. R. (2015). jsPsych: A JavaScript library for creating behavioral experiments in a web browser. Behavior Research Methods, 47(1), 1-12. doi: 10.3758/s13428014-0458-y.

Maxcey, A. M., Shiffrin, R. M., Cousineau D., \& Atkinson, R. C. (submitted). Psychonomic Bulletin \& Review (PBR-BR-21-154).

Rouder, J. N. (2016). The what, why and how of Born-Open Data. Behavior Research Methods, 48, 1062-1069. doi: 10.3758/s13428-015-0630-z

Simmons, J. P., Nelson, L. D., \& Simonsohn, U. (2011). False-positive psychology: Undisclosed flexibility in data collection and analysis allows presenting anything as significant. Psychological science, 22(11), 1359-1366. 


\section{Figure Captions}

Figure 1. Slide triggered by the InitializeBornOpen event.
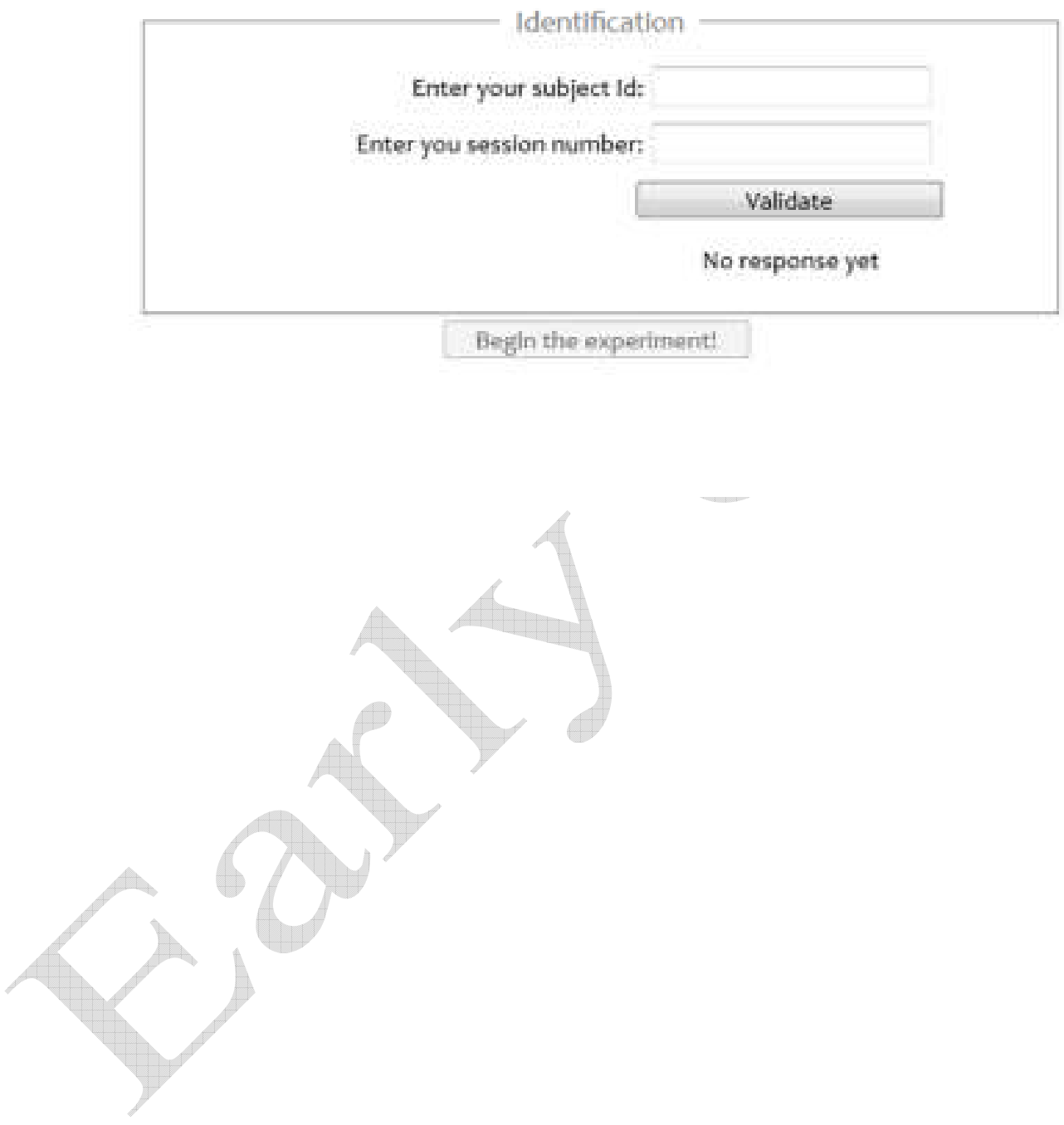


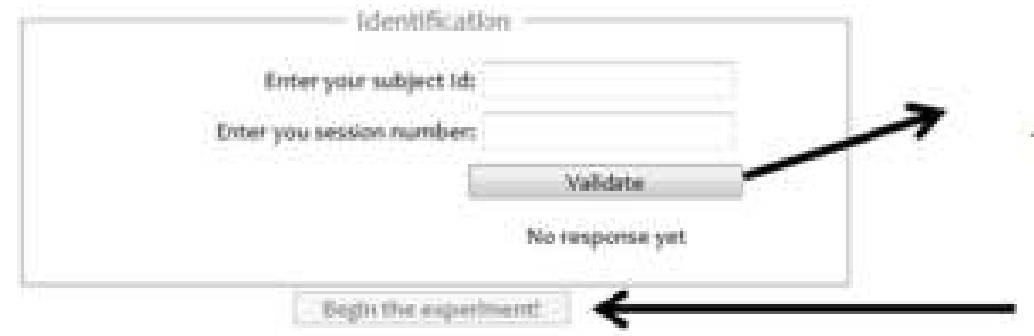

Beghitie expentiont

checkExperimentsubjectSegsion.php verify that the experiment's folder exists .. that the subject and session ID are present in the invitations. $t x \in$ list and that its status is not "done" If this is the case, enable the buttor

an_finish: functionis :

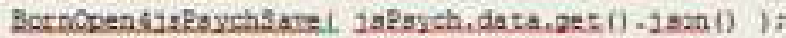

saveFxperimentsubject Session. pho

Save the data on the server

.. and on GitHubOWNER/REPOSITORY

- update subjectsLog. $t x t$ if exists

decrement the number of sessions left

$$
\text { (if not inf) }
$$

... and send emails to owner and participant.

This function retums nothing to jsPsych. 